\title{
Natural Field Orientation Concept: a Tutorial
}

\author{
G. Mirzaeva and R.E. Betz \\ School of Electrical Engineering and Computer Science \\ University of Newcastle, Callaghan, NSW, Australia, 2308
}

\begin{abstract}
Natural Field Orientation (NFO) is a technique for generating a rotating reference frame position for an induction machine which is aligned with the stator flux. The term "natural" is applied because there is an implicit tendency for the rotating frame to realign with the correct stator flux frame position if there is a perturbation away from this position. This paper presents a tutorial on the different forms of NFO and their various merits and deficiencies relative to each other. In addition it also examines the relationship between NFO and stator flux orientation (SFO). It briefly examines frame alignment stability issues in both NFO and SFO, highlighting the essential differences in the performance. Finally, a rotor flux NFO version is developed and compared to the stator flux oriented NFO. The discussions are backed up by simulation and experimental results where appropriate.
\end{abstract}

\section{NOMENCLATURE}

$R_{s}, R_{r}$ - stator and rotor resistances;

$L_{m}, L_{s}, L_{r}$ - magnetising, stator and rotor inductances;

$\sigma$ - leakage coefficient, $\sigma=1-L_{m}^{2} /\left(L_{s} L_{r}\right)$;

$(x, y)$ - axes of the frame aligned with the actual position of the stator flux vector. The $x$ axis is aligned with the stator flux vector;

$(d, q)$ - control frame aligned with the estimated position of the stator flux vector;

$u_{s x}, u_{s y}$ - stator voltage vector projections onto $(x, y)$ axes. Similarly for stator current $i$ and back-emf $e$ vectors;

$u_{s d}, u_{s q}$ - stator voltage vector projections onto $(d, q)$ axes. Similarly for stator current $i$ and back-emf $e$ vectors;

$\left|\underline{i}_{m s}\right|,\left|\underline{\psi}_{s}\right|$ - magnitudes of stator flux and stator magnetising current;

$\left|\underline{i}_{m r}\right|,\left|\underline{\psi}_{r}\right|$ - magnitudes of rotor flux and rotor magnetising current;

$\underline{u}_{s \psi s}$ - the stator voltage space vector in a stator flux frame;

$\underline{i}_{s \psi_{s}}$ - the stator current space vector in a stator flux frame;

$\left|\underline{\psi}_{s}^{*}\right|$ - reference value of the stator flux magnitude. Similarly,

* denotes reference values for other quantities;

$\omega_{m s}, \omega_{m r}$ - angular velocities of stator flux and rotor flux vectors;

$\omega_{r}$ - shaft angular velocity (elec. $\mathrm{rad} / \mathrm{sec}$ ).

\section{INTRODUCTION}

Natural Field Orientation (NFO) concept was first introduced and patented in the 1980 s [1]. In its original version it was closely related to stator flux orientation (SFO). Some authors, for example, in $[2,3]$ refer to it as a simplified open loop method of SFO control. Simplification comes from the fact that the stator flux vector is not estimated but assumed to be at its reference value. Since the NFO algorithm uses the reference flux, it does not have to carry out problematic integrations to find out the correct flux. Assuming that the flux is at a constant value, the stator flux vector will be $90^{\circ}$ spatially lagging the stator flux voltage and can be easily obtained if this voltage is accurately measured.
One of the important properties of SFO is that there is a natural tendency for a misaligned control frame (i.e. when the control frame is not aligned with the stator flux) to implicitly realign. The assumption that the stator flux is at the reference value, which is the fundamental difference between NFO and $\mathrm{SFO}$, does not affect the inheritance of the realignment property from SFO to NFO [4]. This interesting property of NFO together with other positive features like low parameter sensitivity justify more detailed examination of the relative merits of $\mathrm{NFO}$ versus SFO.

The remainder of this paper will consider a family of control techniques, broadly grouped under the umbrella of NFO, with the common feature that the flux magnitude is assumed to be equal to its reference value. Apart from obvious sensor and sensorless options, the NFO family includes current-fed, voltage-fed or mixed (current-fed on one axis and voltage-fed on the other) modifications, as well as stator flux or rotor flux oriented variants. The remainder of this paper will present a brief tutorial on the NFO family of control strategies. Section II will review the raw NFO concept and present some of the key equations. Section III will consider some of the differences between the closely related SFO and NFO. Special emphasis will be placed on issues associated with parameter robustness differences between the two algorithms. Section IV will consider the implications of current fed and voltage fed variants of NFO. Section V will consider the implications of stator flux oriented NFO versus rotor flux oriented NFO. Finally, Section VI will present some conclusions and observations about the NFO algorithm, especially with reference to SFO.

\section{RAW NFO CONCEPT}

In the original patents $[1,5]$ the NFO concept was described as a way of transforming an induction machine into an equivalent voltage fed DC machine, where the voltage on one axis controlled the field and the voltage on the other axis the torque producing current. This way of viewing the NFO concept can be easily developed directly from the stator voltage equation of an induction machine:

$$
\underline{u}_{s \psi s}=R_{s} \underline{i}_{s \psi s}+L_{m} \frac{d}{d t}\left|\underline{i}_{m s}\right|+j \omega_{m s} L_{m}\left|\underline{i}_{m s}\right|
$$

Breaking (1) into real and imaginary parts gives:

$$
\begin{aligned}
& u_{s x}=R_{s} i_{s x}+L_{m} \frac{d\left|\underline{i}_{m s}\right|}{d t} \\
& u_{s y}=R_{s} i_{s y}+\omega_{m s} L_{m}\left|\underline{i}_{m s}\right|
\end{aligned}
$$

Equations (2) and (3) are very close to the equations for a separately excited DC machine with compensating windings using standard notation these can be written as:

$$
\begin{aligned}
& u_{f}=R_{f} i_{f}+L_{f} \frac{d i_{f}}{d t} \text { (the field equation) } \\
& u_{a}=R_{a} i_{a}+\omega_{r} L_{f} i_{f} \text { (the armature equation) }
\end{aligned}
$$




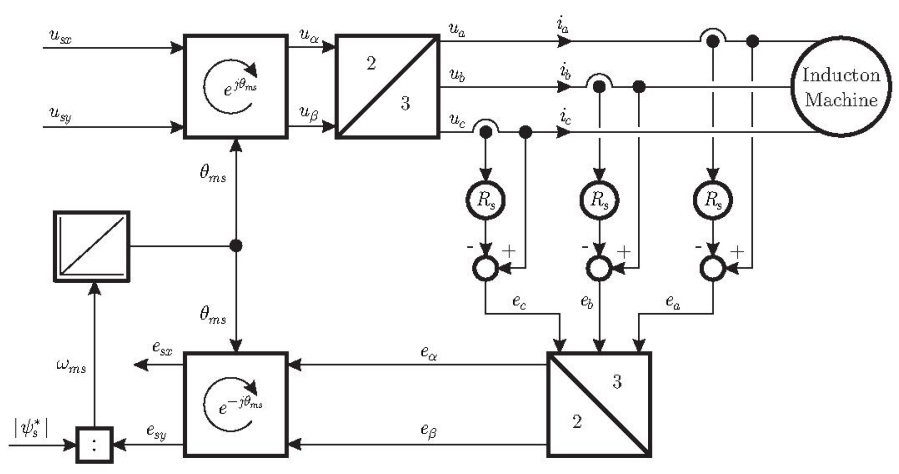

Fig. 1: Raw NFO algorithm that converts an induction machine into a virtual voltage fed DC machine.

The key difference between field equation (2) as compared to (4) is that $\left|\underline{i}_{m s}\right|$ is a function of not only the $i_{s x}$ current but also of the rotor conditions and $i_{s y}$ (via cross-coupling effects). These various parameters are reflected in the flux of the machine via the expression $\psi_{s}=\int e_{s x} d t=\int\left(u_{s x}-R_{s} i_{s x}\right) d t$. Therefore if the machine is voltage driven and if the $i_{s x}$ component of current is measured and the stator resistance $R_{s}$ is accurately known, then a $u_{s x}$ voltage can be determined so that the desired value of $\left|\underline{i}_{m s}\right|$, and therefore flux, is obtained in the machine.

If one then applies the NFO concept we have that:

$$
\omega_{m s}=\frac{u_{s y}-R_{s} i_{s y}}{L_{m}\left|\underline{i}_{m s}^{*}\right|}=\frac{e_{s y}}{\left|\underline{\psi}_{s}^{*}\right|}
$$

where $\left|\underline{i}_{m s}^{*}\right|$ and $\left|\underline{\psi}_{s}^{*}\right|$ denote the reference values for these quantities and not their actual values. Another observation is that in steady state, when $\left.d\right|_{m s} \mid / d t=0$ the back-emf voltage $e_{s x}=u_{s x}-R_{s} i_{s x}$ should equal zero. Figure 1 shows the structure of the raw NFO algorithm more or less as it appeared in the original patents.

\section{NFO VERSUS TRADITIONAL SFO}

As mentioned in the introduction, NFO is a special form of the traditional SFO algorithm. The main difference between the SFO and NFO techniques is shown in Figures $2 a$ and $2 b$ respectively. These diagrams show the relevant parts of the SFO and $\mathrm{NFO}$ algorithms related to the generation of the stator flux vector position in the machine. One can see that NFO has no integration to determine the flux of the machine, while with SFO an estimate of the stator flux magnitude is obtained by integrating the $e_{s x}$ component of the stator voltage vector.

One obvious and significant advantage of NFO, as compared to $\mathrm{SFO}$, is its improved frame angle robustness to inaccurate knowledge of the stator resistance. In SFO if the stator resistance has, for example, a lower value in the controller than the true value in the machine, then the $e_{d s}$ voltage will be zero under "steady state" but the true $e_{s x}$ back-emf within the machine will be non-zero. Consequently the flux in the machine will be building up, undetected by the control algorithm. This will result in a tendency for the calculated frame angular velocity to increase since the $e_{s y}$ value will increase, but the estimated flux magnitude will remain the same. Fortunately, under current fed control strategies, the effect will stabilise at some significant non-zero frame misalignment angle when the flux producing current will be low enough to compensate for these other effects.

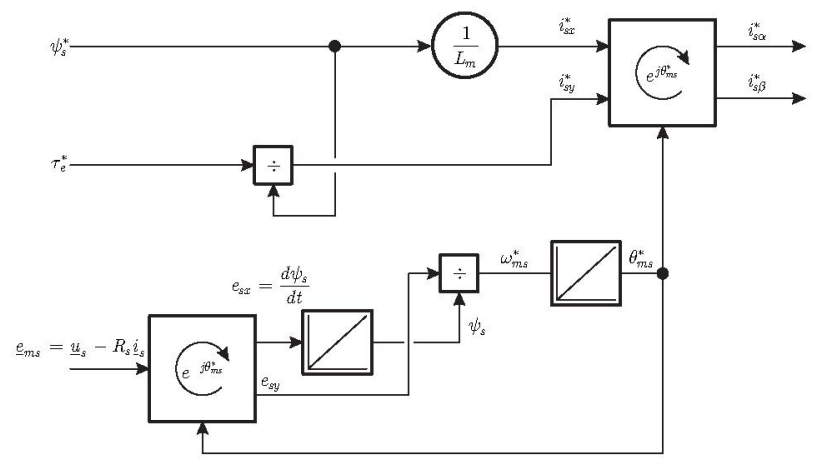

(a) Traditional stator flux orientation

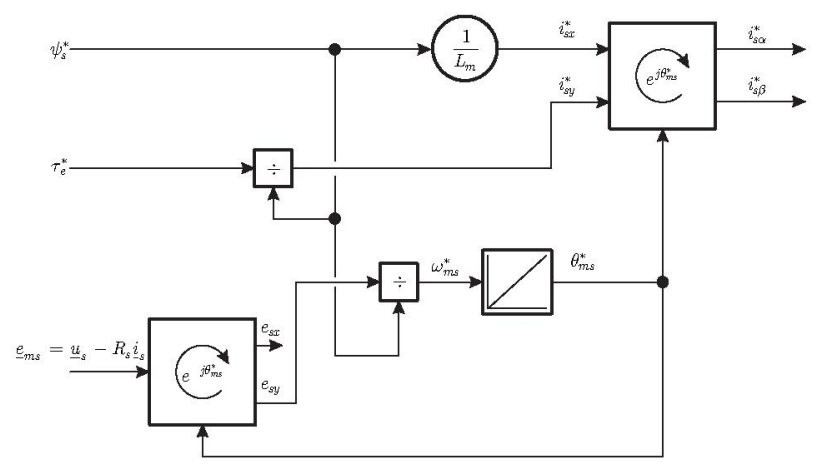

(b) Natural field orientation

Fig. 2: Comparison between traditional SFO and NFO (both current fed, leakage is ignored).

Errors in the stator resistance will also have an effect on NFO. However, since we are not integrating the flux producing back-emf the precise phenomena described above does not occur. In this case the $e_{s y}$ voltage will be in error because of the resistance error. However, if the machine is operating at a reasonable speed the relative error due to this will be small, and consequently the frame misalignment will be small. In addition to this, for a current fed machine, the error in the frame position is such that it will make the value of $e_{s y}$ change in a fashion to prevent any accumulation of the frame error [68]. This conclusion is supported by the results of the currentfed NFO simulations that appear in Figure 3. These figures show the results of a simulation where the induction machine is operating as a NFO controlled torque source (i.e. no speed or position feedback). In the first case the correct $R_{s}$ value is used by the algorithm. In the second case an error of more than $25 \%$ is introduced in $R_{s}$. One can see that performance of the algorithm is only marginally worse in the second case than in the first, which indicates that the frame position is not grossly in error [6].

Figure 4 shows simulation and experimental results for the raw (unaugmented) NFO algorithm with $50 \% R_{s}$ error. The NFO algorithm has a speed controller around it, and the desired speed is a simple step. The simulation in this case was very accurate and included the inverter dead-time. One can see that the experimental and simulation results are very close, and that a static $e_{s d}$ voltage appears indicating a static frame misalignment to compensate for the $R_{s}$ error. Numerous other experiments have been carried out which confirm the robustness of NFO with respect to $R_{s}$ error [6-8].

Similary Figure 5 shows the performance of SFO under the same parameter inaccuracy in $R_{s}$, and with all other parameters known exactly. The algoirthm in implemented open 


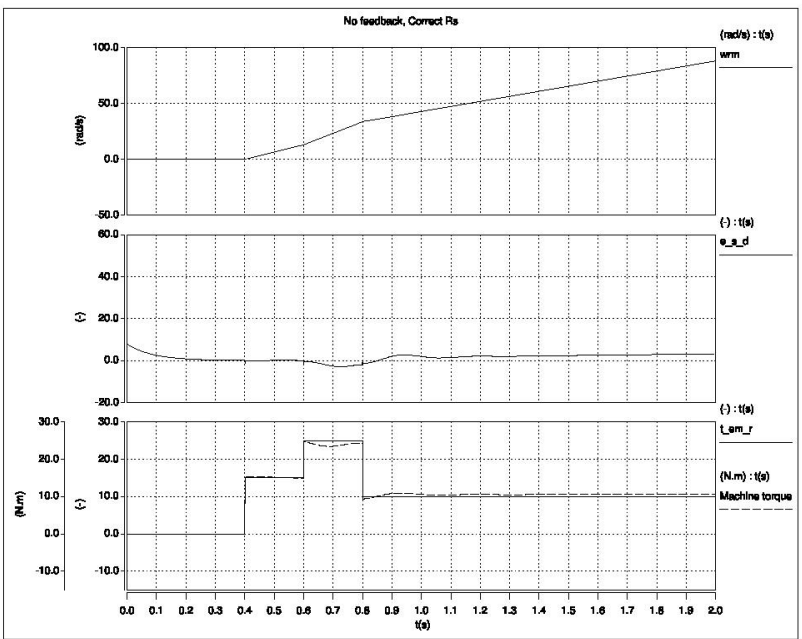

(a) Correct value: $R_{s}=0.435 \Omega$

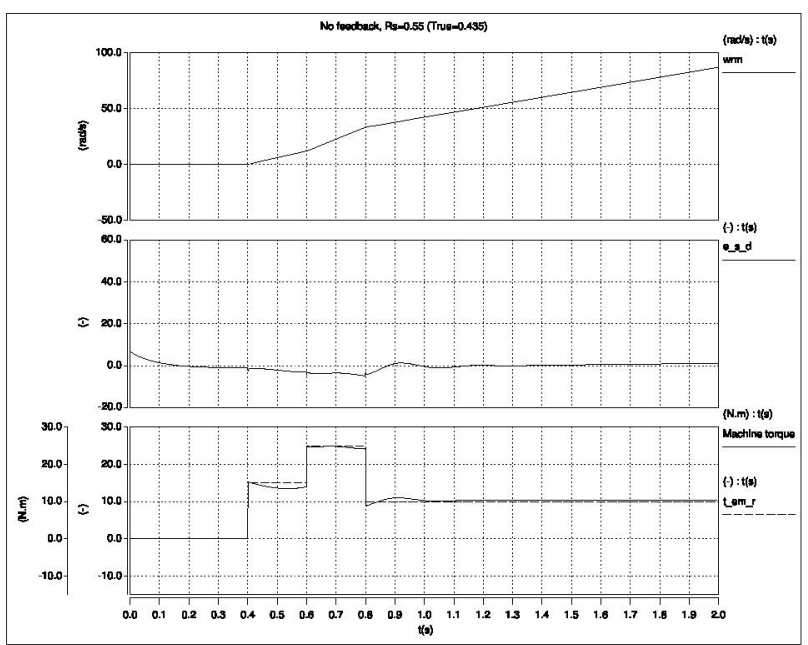

(b) Erroneous value: $R_{s}=0.55 \Omega$

Fig. 3: Simulation of the current fed NFO algorithm.

loop (i.e. without a flux feedback path), so that the situation is equivalent to the conditions under which the NFO algorithm was operating. As can be seen, the error in the stator resistance leads to totally unacceptable torque performance in the SFO algorithm. Similar results are obtained if there is a flux feedback loop with SFO.

Whilst SFO is more susceptible to frame misalignment from inaccurate $R_{s}$ knowledge as compared to $\mathrm{NFO}, \mathrm{SFO}$ has the advantage that it does not have the same problem with unstable frame alignment in regeneration $[4,6,7]$. The NFO algorithm in its original form demonstrates static frame alignment instability over virtually the whole regeneration region. This means that under regeneration any error in the NFO control frame position will tend to lead to an increase in the error. For example, if the control frame moves ahead of the correct frame position, then under certain conditions the $e_{s y}$ value can become larger than it should be under perfect alignment. This results in the $\omega_{m s}$ estimated in equation (6) being larger than the correct angular velocity value, and consequently the control frame will move further away from the "true" frame position. This clearly will lead to unacceptable performance. A simulation of the "raw" NFO algorithm in motoring and regeneration is shown in Figure 6. It shows that the machines performance is excellent under motoring, but when the torque reference

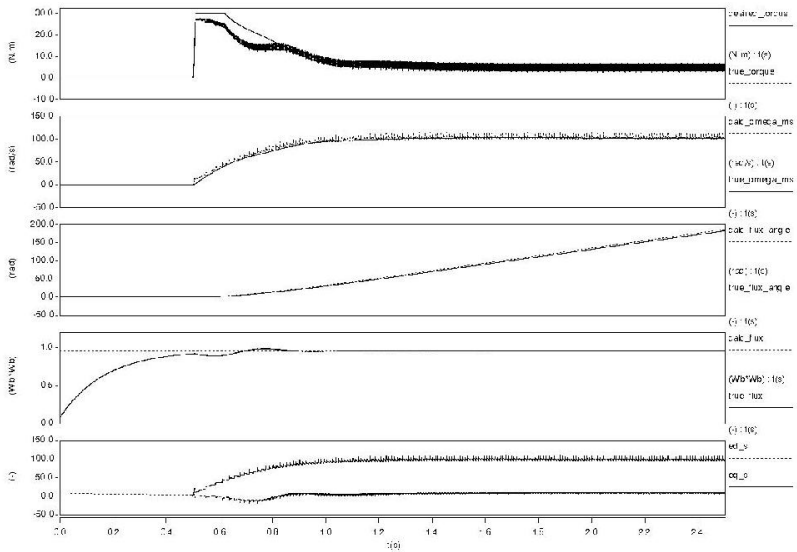

(a) Simulation data
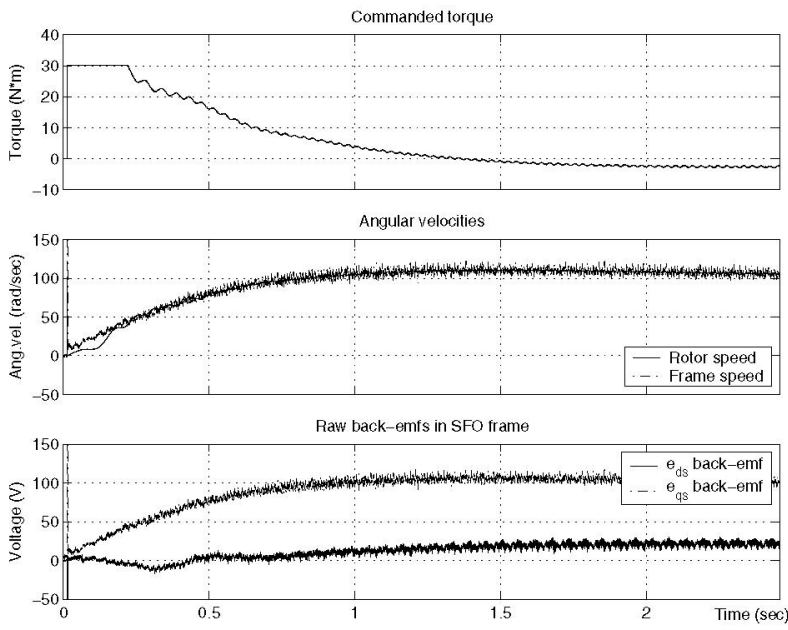

(b) Experimental data

Fig. 4: NFO test: Unaugmented, $\Delta R_{s}=+50 \% R_{s}, L_{m}$ correct

becomes negative the machine torque initially follows it, but then collapses to zero as the control frame slides away from the true frame position.

The frame instability and misalignment can be corrected by augmenting (6) using the $e_{s d}$ value as follows $[4,6]$ :

$$
\omega_{m s}=\frac{e_{s q}-k\left[\operatorname{sign}\left(e_{s q}\right) e_{s d}\right]}{\left|\underline{\psi}_{s}^{*}\right|}
$$

where $k$ is a tunable control parameter. Figure 7 shows the performance of NFO using this approach. Extensive simulation experimental results show that this augmentation makes the algorithm more robust to parameter errors.

Let us consider SFO under regeneration. The $d q$ frame is assumed to be ahead of the $x y$ frame, which means that the angular velocity of the $d q$ frame is higher than the $x y$ frame. Assuming a current fed machine, the frame misalignment results in an increase in the $e_{s d}$ (away from the ideal zero value). This voltage is integrated to calculate an estimate of the flux, which is used in equation (6) (in place of the reference flux). The value of $e_{s q}$ is a projection of $e_{s y}$, and therefore is less than $e_{s y}$. Furthermore, the projection of $i_{s d}$ onto the $x$ axis is also less, and this means that the actual flux magnitude will decrease from the desired flux. The net result of all these influences is that the new value of the $d q$ frame angular velocity will decrease. The feedback effect of the stator flux estimate is strong enough to force the angle error between the two frames 


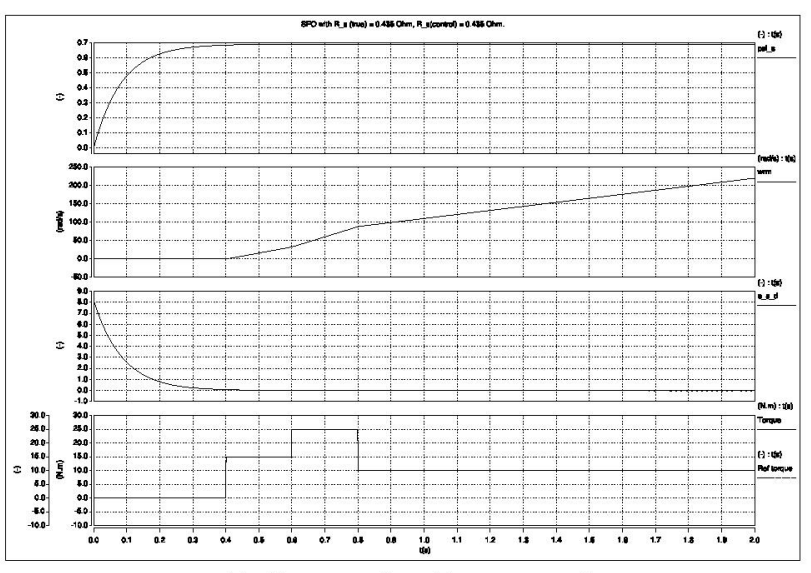

(a) Correct value: $R_{s}=0.435 \Omega$

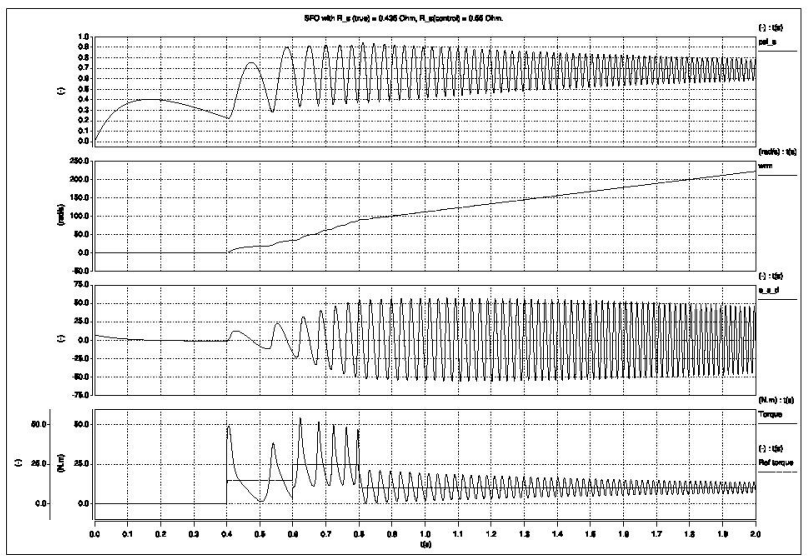

(b) Erroneous value: $R_{s}=0.55 \Omega$

Fig. 5: Simulation of current fed SFO algorithm.

to zero. Figure 8 shows the performance of SFO under similar conditions to those shown for NFO in Figure 6. The parameters of the machine used in the SFO controller are the true machine parameters in this example. As can be seen SFO performs very well in both motoring and regeneration modes.

Remark 1: It turns out the SFO benefits substantially by the use of $e_{s d}$ feedback augmentation [6].

Whilst NFO is robust, relative to SFO, with respect to $R_{s}$ errors, it is more susceptible to errors in $L_{m}$. Errors in $L_{m}$ again lead to errors in the flux produced in the machine because the reference current is calculated using the $L_{m}$ value as shown in Figure $2 \mathrm{~b}$. A lower reference current results in a lower $e_{s y}$ value which in turn feeds around to the algorithm via $\underline{e}_{m s}$. It can be shown $[6,8]$ that NFO can tolerate $\pm 15 \%$ errors in the $L_{m}$ and still work satisfactorily. Fortunately standard machine parameter tests are able to satisfy this accuracy in this parameter. SFO on the other hand in its practical implementation, tends not to be as sensitive to $L_{m}$ error. Because an estimate of the actual flux, calculated by voltage integration, is available in this algorithm, then a feedback loop can be simply implemented to correct flux errors. This process of course, is precisely why the algorithm is sensitive to $R_{s}$.

Inverter dead-time can also have an influence on the performance of NFO since it affects the accuracy of the determination of the machine back-emf. It can be shown that the effect of dead-time is very similar to resistance error, and results in a misalignment of the control frame with respect to the true stator flux frame. Investigation of the influence of dead-time has shown that the robustness of the NFO to its effects is similar

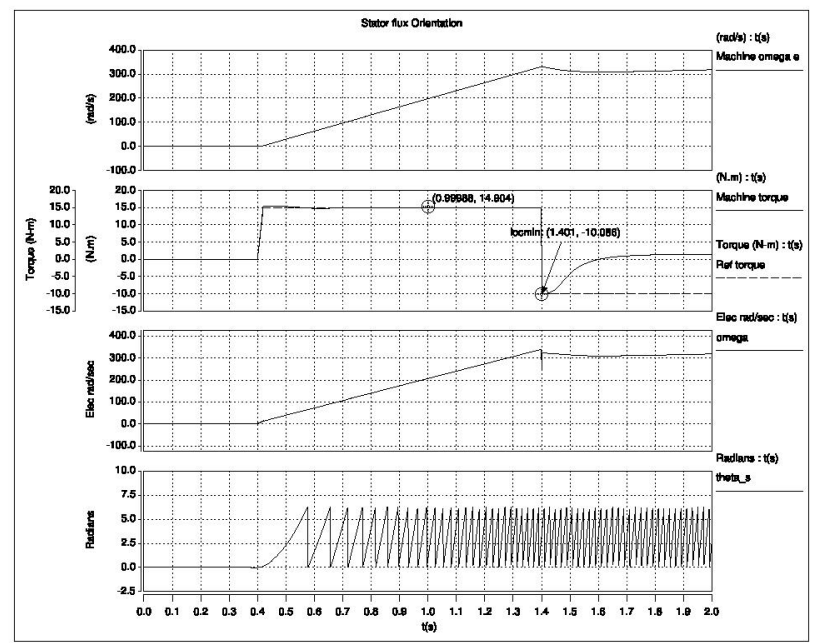

Fig. 6: Stator flux oriented NFO for motoring and regeneration.

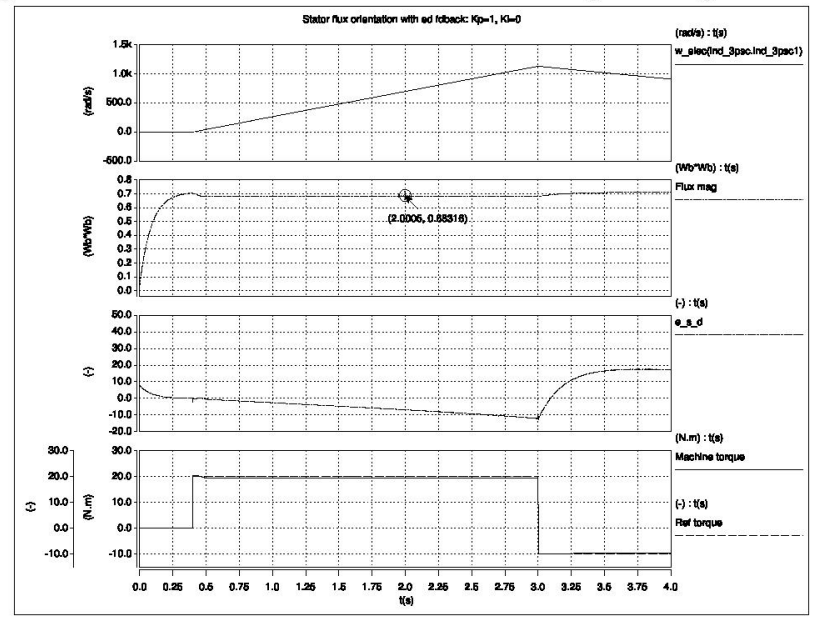

Fig. 7: NFO simulation with $k=1$ with $e_{s d}$ feedback.

to its robustness with respect to stator resistance error.

Summary 1: The following interim observations can be made:

- NFO is essentially an alternative form of SFO.

- NFO has the disadvantage that it is statically unstable with respect to frame alignment in regeneration.

- Because NFO does not use integration to estimate the stator flux, it is particularly robust with respect to errors in the stator resistance and applied voltage errors due to deadtime effects. This also means that it can operate at lower speeds as compared to the traditional SFO algorithm.

- NFO is more sensitive to $L_{m}$ error than SFO, but the level of sensitivity is not high and is not an impairment to practical implementation.

Remark 2: Note that orientation of NFO to the stator flux vector brings the same limitations as traditional vector control aligned to this frame, namely that there is effectively a static torque overload limit caused by the machine effectively slipping over the top of the torque slip characteristic [9].

\section{I-I, V-I AND V-V IMPLEMENTATIONS OF NFO}

Equations (2) and (3) suggest several alternative control topologies. So far we have considered the strategy that uses current control on each axis of the machine (we shall denote this as $i-i$ control). This is the same control scheme used by 


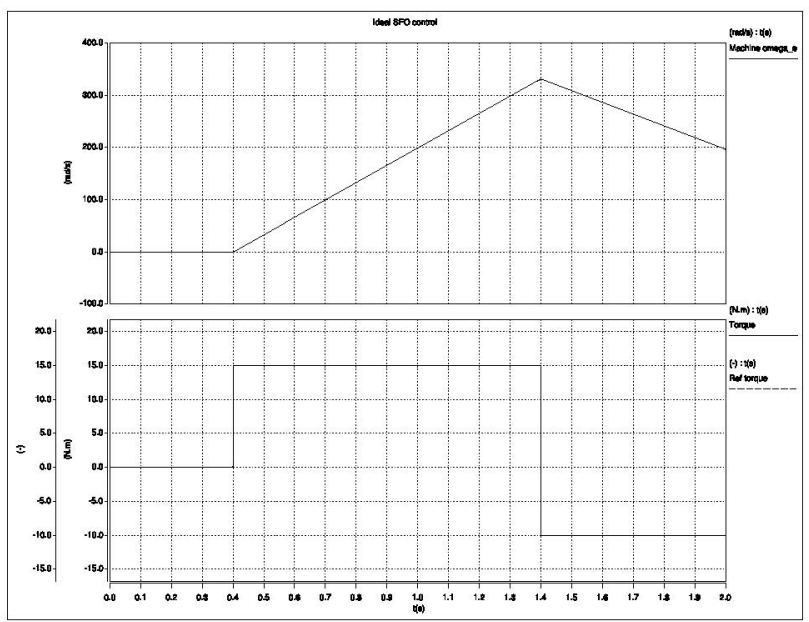

Fig. 8: Simulation of SFO under motoring and regeneration conditions.

most field oriented vector controllers. A block diagram of the i-i control with stator flux oriented NFO appears in Figure 9a. It is implemented by using current control loops around both flux producing and torque producing axes of the machine. Effectively the machine is being driven by current sources. Two other alternatives are to use voltage control on both axes (denoted as $\mathrm{V}-\mathrm{v}$ control), or current control on one axis and voltage control on the other (denoted as $\mathrm{v}$-i control). NFO with $\mathrm{v}-\mathrm{V}$ control was suggested in $[1,2,5,10]$ and NFO with $\mathrm{v}-\mathrm{i}$ control in [11].

In $v-i$ control it is natural to use voltage control on the flux producing axis of the machine since flux and voltage are related via Faraday's Law, and current control on the torque axis since torque is related to the current magnitude orthogonal to the flux vector. A block diagram of the $\mathrm{v}-\mathrm{i}$ control used in the simulations is shown in Figure 10a. To simulate $v-v$ control one can use the same scheme after removing the explicit control loop around the current (the torque loop).

Both $\mathrm{v}-\mathrm{v}$ and $\mathrm{v}-\mathrm{i}$ schemes showed reasonable performance when the $R_{s}$ value was precisely known. However, with the same erroneous $R_{s}$ value as that used in the simulation of the i-i NFO (see Figure 3b), both v-i and v-v control schemes were found to be completely unusable. Simulation results for the $v-i$ NFO under this condition appear in Figure 10b.

Remark 3: In the $\mathbf{i}-\mathbf{i}$ driven case we are applying current in such a manner that $i_{s d}=i_{s d}^{*}$. Then we measure voltage $u_{s d}$ and estimate $e_{s d}=u_{s d}-R_{s} i_{s d}$. If there are errors in $R_{s}$ which cause the control frame misalignment, a non-zero value of the $e_{s d}$ would clearly indicate this misalignment. Indeed the presence of $e_{s d}$ will detect misalignment regardless of the cause. This can be used to compensate for it by applying, for example, an additional stabilisation loop based on the $e_{s d}$ signal. This idea will be explored in depth in a companion paper [12].

Remark 4: In the $\mathrm{v}-\mathrm{i} / \mathrm{v}-\mathrm{v}$ situation if misalignment occurs (for whatever reason) there is no indication of this via the $e_{s d}$ voltage - since we are applying $u_{s d}$ in such a manner that $e_{s d}=0$. In other words there is an implicit assumption that one has perfect alignment of the $d q$ and $x y$ axes. There is circularity in the calculation of $e_{s d}$ - i.e. we are applying $u_{s d}=i_{s d}^{*} R_{s}$ relative to the control frame $d q$ axes and then simply measuring the same applied voltage to the same axes. Errors in $R_{s}$ will result in $e_{s d} \neq e_{s x}$, and therefore the flux loop will calculate

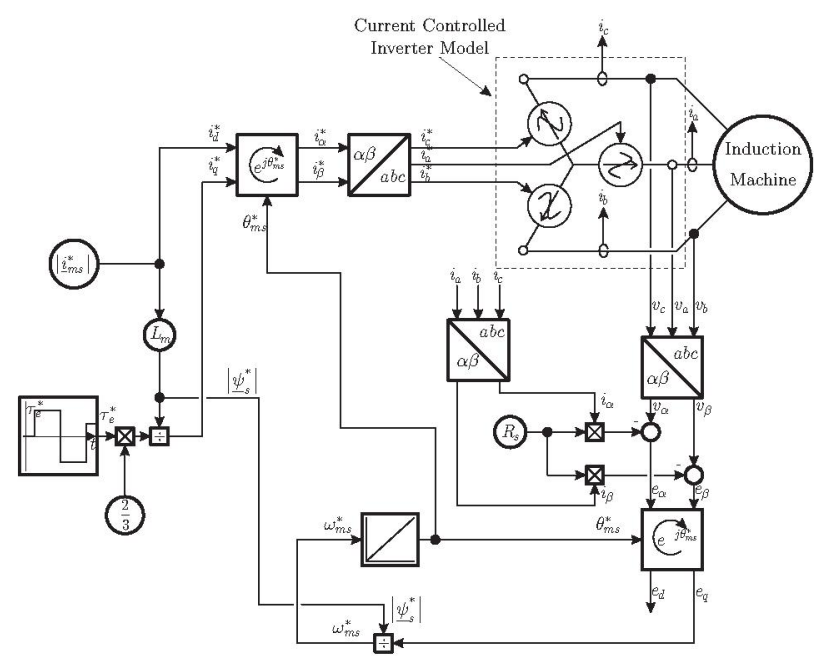

(a) Stator flux oriented control

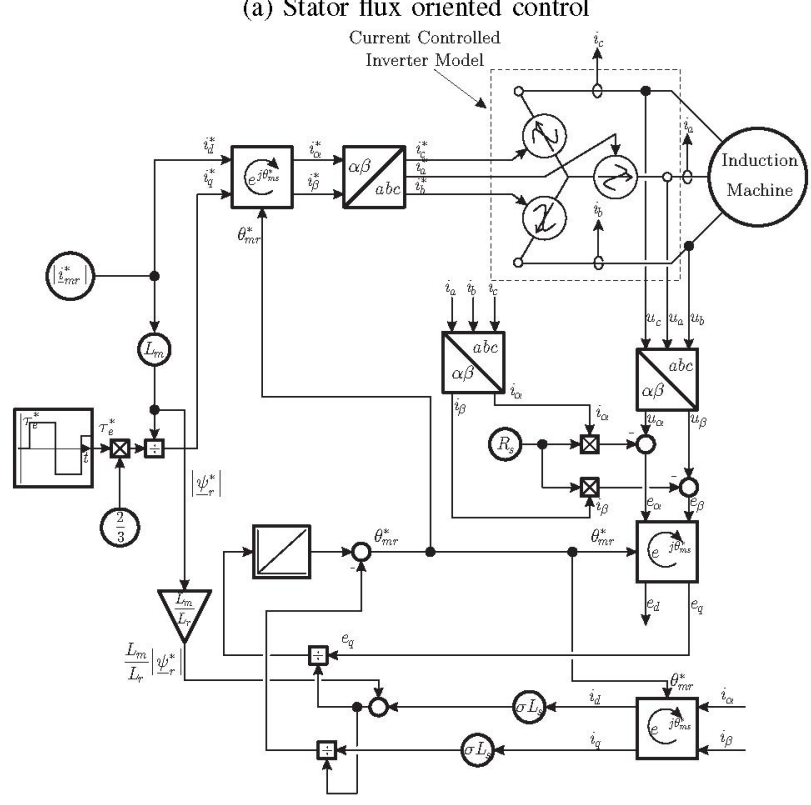

(b) Rotor flux oriented control

Fig. 9: Simulation of the current fed (i-i) NFO control algorithm.

an incorrect flux. Therefore if there are errors in $R_{s}$ which cause misalignment this misalignment cannot be compensated for with the $e_{s d}$ feedback signal as in the $\mathrm{i}-\mathrm{i}$ case. From this point of view, the $\mathrm{i}-\mathrm{i}$ control scheme would be a preferred option if $R_{s}$ is not adjusted during the machine's operation.

\section{STATOR VERSUS ROTOR FLUX ORIENTATION NFO}

The torque limitation of the stator flux vector control mentioned in Remark 2 can be overcome if the NFO aligns with the rotor flux vector. In this case the torque overload is limited only by the ability of the windings to conduct the large overload currents. Consider the stator voltage equations in a rotor flux oriented frame:

$$
\begin{aligned}
& u_{s x}=R_{s} i_{s x}+\sigma L_{s} \frac{d i_{s x}}{d t}+\frac{L_{m}^{2}}{L_{r}} \frac{d\left|\underline{i}_{m r}\right|}{d t}-\sigma L_{s} \omega_{m r} i_{s y} \\
& u_{s y}=R_{s} i_{s y}+\sigma L_{s} \frac{d i_{s y}}{d t}+\sigma L_{s} \omega_{m r} i_{s x}+\omega_{m r} \frac{L_{m}^{2}}{L_{r}}\left|\underline{i}_{m r}\right|
\end{aligned}
$$

These equations are much more complex than (2) and (3). From equation (9) and with the NFO concept applied the angular 


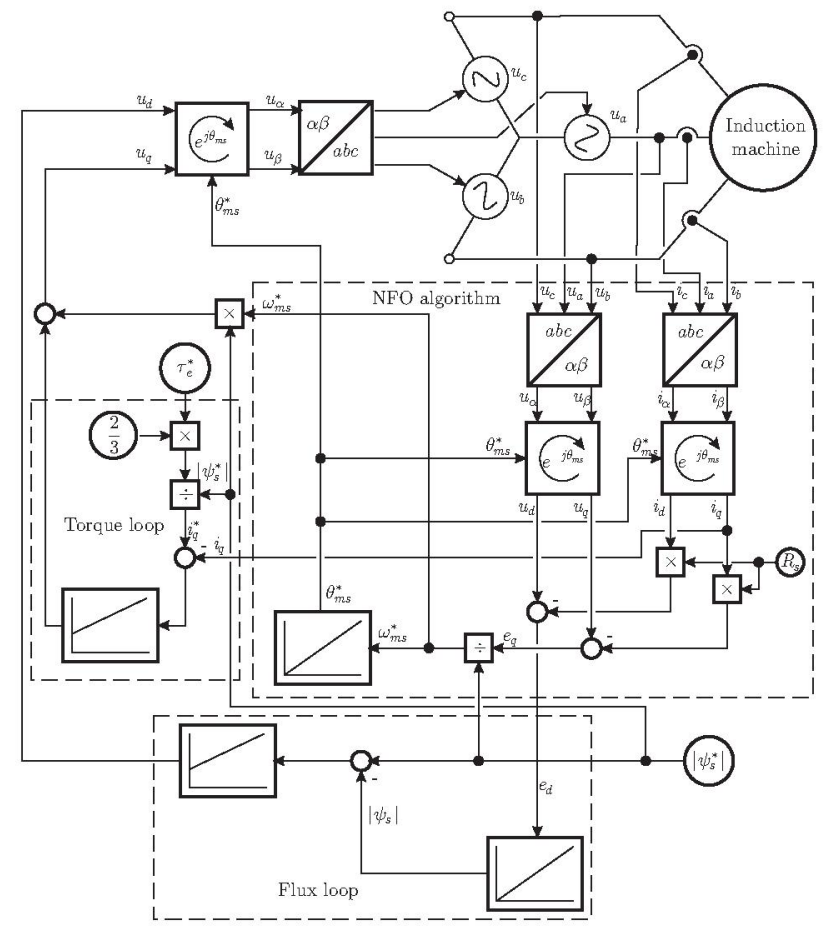

(a) Block diagram of $\mathrm{v}-\mathrm{i}$ NFO control

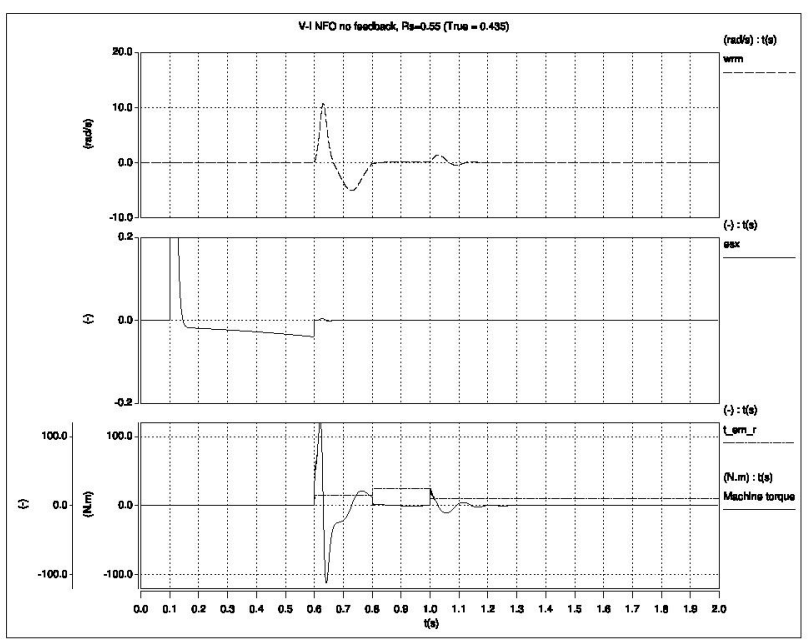

(b) Simulation results with erroneous $R_{s}=0.55 \Omega$

Fig. 10: Simulation of the v-i NFO control algorithm.

velocity of the rotor flux vector can be estimated as:

$$
\omega_{m r}=\frac{u_{s y}-R_{s} i_{s y}-\sigma L_{s} \frac{d i_{s y}}{d t}}{\frac{L_{m}}{L_{r}}\left|\underline{\psi}_{r}^{*}\right|+\sigma L_{s} i_{s x}}
$$

which implies a trickier integration to obtain the frame angular position than with the respective expression (6) for the stator flux orientation. From equation (8) it is clear that in steady state $e_{s x}=u_{s x}-R_{s} i_{s x}=-\sigma L_{s} \omega_{m r} i_{s y}$. This is a non-zero but still a relatively small value. So, similarly to the SFO version, nonzero $e_{s x}$ values should mainly indicate frame misalignment. A block diagram of the rotor flux oriented NFO algorithm is shown in Figure 9b. This scheme was used in simulations and showed results close to that for the SFO control with the advantage of not being limited in maximum torque.

Remark 5: The rotor flux oriented NFO control retains the advantages of the stator flux oriented NFO control, such as low sensitivity to $R_{s}$ value and indication of the frame misalignment by a non-zero $e_{s x}$ value. At the same time, it requires more parameters $\left(\sigma L_{s}\right)$ for its implementation. In some applications the latter could be an appropriate trade-off for overcoming the pull-out torque limit implied by the stator flux oriented control.

Remark 6: Simulation results of the rotor flux oriented version of NFO are very similar to those obtained for stator flux oriented NFO. The rotor flux variant of the algorithm still has the same problem with static frame instability in regeneration as the stator flux variant.

\section{CONCLUSIONS}

The main observations and conclusions from this paper are:

- NFO is a variant of SFO. NFO is much more robust with respect to errors in $R_{s}$ as compared to SFO.

- Raw NFO has static reference frame instability in regeneration. This can be corrected by using $e_{s d}$ in a feedback loop.

- The NFO variants for the current-fed, voltage-fed and voltage-current-fed induction machine are discussed and compared. The $i-i$ version of NFO has the advantage that $e_{s d}$ can be used as a feedback signal to correct frame instability and frame misalignment due to parameter errors. The $\mathrm{v}-\mathrm{v}$ and $\mathrm{v}-\mathrm{i}$ forms of NFO are very sensitive to $R_{s}$ accuracy, and cannot use $e_{s d}$ feedback.

- The stator flux version of NFO has a static torque limit. The rotor flux version overcomes this torque limit at the cost of more parameter dependence.

\section{REFERENCES}

[1] R. Jönsson, "Method and apparatus for controlling an AC motor," United States Patent 4,458,193, July 1984.

[2] M. Kazmierkowski, M. Malinowski, D. Sobczuk, F. Blaabjerg, and J. Pedersen, "Simplified stator flux oriented control," in Proceedings of the IEEE International Symposium on Industrial Electronics, ISIE'99, vol. 2, 1999 , pp. 474479.

[3] G. S. Buja and M. P. Kazmierkowski, "Direct torque control of pwm inverter-fed ac motors - a survey," IEEE Transations on Industrial Electronics, vol. 51, no. 4, pp. 744-757, August 2004.

[4] R. Betz and G. Mirzaeva, "Frame alignment stability issues in natural field orientation," in CDROM Proceedings of the IEEE IAS Annual Meeting. IEEE, October 2006.

[5] R. Jönsson, "Method and apparatus for controlling an ac induction motor by indirect measurement of the air-gap voltage," United States Patent 5,294,876, March 1994 .

[6] R. E. Betz, G. Mirzaeva, and D. Pulle, "Frame alignment stability issues in natural field orientation," School of Electrical Engineering and Computer Science, University of Newcastle, Australia, Tech. Rep. V1.13, January 2006.

[7] G. Mirzaeva and R. Betz, "Analysis of frame alignment issues in natural field orientation including non-linear and leakage inductance effects," September 2007, submitted to EPE2007.

[8] — "Parameter sensitivity issues in natural field orientation," September 2007, submitted to the IEEE IAS Annual Meeting.

[9] P. Vas, Vector Control of AC Machines. Oxford University Press, 1990, ISBN:0-19-859370-8.

[10] R. Jönsson and W. Leonhard, "Control of an induction motor without a mechanical sensor, based on the principle of natural field orientation (NFO)," in International Power Electronics Conference, IPEC'95. IEEJ, April 1995, pp. $1-6$.

[11] D. Pulle, "Analysis of the nfo pm type drive," July 2001, private Report.

[12] G. Mirzaeva and R. Betz, "An improved natural field orientation control of a current fed induction machine," May 2007, submitted to IEMDC2007. 